\title{
INTEGRATED CARE Love in the time of COVID-19: Social prescribing and the paradox of isolation
}

\author{
Authors: Michael Dixon ${ }^{A}$ and Dean Ornish ${ }^{B}$
}

KEYWORDS: COVID-19, prescribing, isolation

DOI:10.7861/fhj.2021-0009

\section{Introduction}

At the time of writing, over 100 million cases of COVID-19 have been reported worldwide, and over 3.7 million in the U.K. ${ }^{1}$ Over 2 million people have died from the disease including over 100,000 in the U.K. ${ }^{2}$ Fortunately, several vaccines have now been developed, but it will be many months before a sufficient number of the population has been inoculated to achieve effective herd immunity. ${ }^{3}$

In the meantime, most efforts have focused on reducing exposure to SARS-CoV-2, which, of course, is vital: staying at home, social distancing, hand washing, wearing gloves, wearing masks and disinfecting.

However, very little has been written about the other half of the equation: how to enhance immune function and overall health so that, if you are exposed to COVID-19, you can potentially reduce the risk of becoming seriously unwell and therefore reduce the death rate.

Most of the mortality from COVID-19 has occurred in people who have one or more chronic diseases. ${ }^{4}$ Most physicians are trained to view coronary heart disease, type two diabetes, earlystage prostate cancer, breast cancer, colon cancer, autoimmune diseases, hypertension, dementia and other chronic diseases as fundamentally different illnesses, with different aetiologies, requiring different treatments.

However, it is becoming increasingly clear that these and many other chronic diseases have more in common than might be expected, as many share similar biological mechanisms, all of which are powerfully affected by the lifestyle choices made every day, for better and for worse. These include:

Authors: Ageneral practitioner, Guy's Hospital, London, UK, chair, College of Medicine and Integrated Health, London, UK and chair, Institute for Social Prescribing - South West Academic Health Science

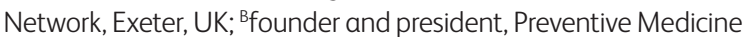
Research Institute, San Francisco, USA
$>$ chronic inflammation

$>$ immune system dysfunction

> chronic emotional stress and overstimulation of the sympathetic nervous system

$>$ lack of sleep

$>$ changes in gene expression and sirtuins

$>$ accelerated shortening of telomeres

$>$ relative preponderance of beneficial to harmful organisms in the microbiome

> increased oxidative stress, deranged cellular metabolism and accelerated apoptosis

$>$ dysfunctions in angiogenesis

$>$ vascular stasis. $^{5}$

Therefore, making lifestyle changes to prevent or induce remission of chronic diseases could hypothetically reduce the risk of death from COVID-19.

$>$ A whole-foods, plant-based diet: low in animal protein, fat, sugar and refined carbohydrates, and high in fruits, vegetables, whole grains, legumes and soy products in unprocessed forms. ${ }^{6}$

> Adequate physical activity: such as walking and strength training.

> Stress reduction: including meditation, gentle yoga-based stretching and breathing techniques that can enable you to do more and stress less. ${ }^{8}$

> Social connections, social support and community. ${ }^{9}$

In short: eat well, move more, stress less and love more.

These lifestyle changes have a positive impact on reducing the risk of developing chronic diseases and may also act to reduce the severity of, or reverse, established disease.

The European Prospective Investigation into Cancer and Nutrition (EPIC) study of nearly 25,000 men and women found that the same lifestyle choices had a large impact on a variety of chronic diseases. Those with four healthy lifestyle factors had a $78 \%$ lower risk of developing any chronic disease. The lifestyle factors included moderate exercise of at least 30 minutes per day; not smoking; normal weight; and a high intake of fruits, vegetables, and whole grains and low meat consumption. When specific diseases were considered, the study reported that those with four healthy lifestyle factors had a $93 \%$ lower risk of getting type 2 diabetes, an $81 \%$ reduced risk of a heart attack, a $50 \%$ lower risk of a stroke and a $36 \%$ reduction in all forms of cancer. ${ }^{10}$ It's worth emphasising that, although the prevalence of type 2 diabetes is high in both Europe and America, this study 
demonstrated that at least $93 \%$ of diabetes is completely preventable.

In 2018, a large-scale study of over 100,000 patients from the Harvard School of Public Health found that those individuals with five healthy lifestyle habits had an $82 \%$ lower risk of dying from cardiovascular disease, a $65 \%$ lower chance of dying from cancer and a $74 \%$ lower risk of dying from all causes during follow-up.

Each of the five lifestyle factors included in the study (diet, smoking, physical activity, alcohol consumption and weight) showed a significant association with increased premature death from all causes, from cancer and from heart disease. Those who were not overweight, never smoked, exercised an average of 30 minutes per day, didn't drink to excess, and ate a healthy diet lived an average of 12-14 years longer. Indeed, each individual factor had a positive effect. ${ }^{11}$

The impact that modifying lifestyle factors may have on viral respiratory diseases is outlined.

\section{Love more}

After 100 years of research on man and animals, psychological stress is considered as a potential cofactor in the pathogenesis of infectious disease. ${ }^{12}$

Staying at home and social distancing help prevent transmission of SARS-CoV-2 but, paradoxically, feeling lonely for prolonged periods can be harmful to one's health. Multiple studies have shown that people who are lonely and isolated are 3-10 times more likely to become ill and die prematurely of virtually all causes when compared with those who have a strong sense of love and community. ${ }^{9,13}$

Also, spreading fear about the coronavirus may, ironically, increase the risk of contracting it. Fear can be self-fulfilling to the extent that it suppresses immune function and makes someone more likely to get the very disease they're most afraid of. ${ }^{14}$

Fortunately, there are many things an individual can do to enhance social connections and, thus, immune function. While it is vitally important to follow the lockdown restrictions imposed by the UK government, it is also wise to spend more time socialising with household members as well using video technologies or just talking on the phone to virtually spend time with friends and family in other places.

Sheldon Cohen is the Robert E Doherty university professor of psychology at Carnegie Mellon University and director of the Laboratory for the Study of Stress, Immunity and Disease; he reported the results of a study in which he dripped rhinovirus (which causes the common cold) in the noses of volunteers. All of them became infected, but not everyone developed the signs and symptoms of a cold.

Those with more types of social ties were less susceptible to common colds, produced less mucus, were more effective in ciliary clearance of their nasal passages and shed less virus. These relationships were unaltered by statistical controls for pre-challenge virus-specific antibody, virus type, age, sex, season, body mass index, education and race. Susceptibility to colds decreased in a doseresponse manner with increased diversity of the social network. There was an adjusted relative risk of 4.2 comparing people with fewest (one to three) to those with most (six or more) types of social ties. ${ }^{15}$

In other words, those that had only one to three social ties (defined as a phone call or visit from a friend every 2 weeks) were 4.2 times more likely to develop the signs and symptoms of a cold than those with six or more social ties during that time.

\section{Stress less}

In an earlier study, Cohen dripped five types of respiratory viruses (rhinovirus types 2, 9 or 14, respiratory syncytial virus or coronavirus type $229 \mathrm{E}$ (a less deadly form than the current coronavirus)) into volunteers. The rates of both respiratory infection and clinical colds increased significantly in a dose-response manner with increases in the degree of psychological stress. Infection rates ranged from approximately $74 \%$ to approximately $90 \%$, according to levels of psychological stress, and the incidence of clinical colds ranged from approximately $27 \%$ to $47 \%{ }^{16}$

In other words, stress suppressed their immune function. Because of this, the more stressed they felt, the more likely they were to develop the signs and symptoms of a respiratory infection.

And in a more recent study, he found that those who he infected with rhinoviruses who experienced positive emotions had a 2.9 times lower risk of developing a cold than those who did not, in a dose-response fashion. Thus, feeling happy and peaceful enhances immune function. ${ }^{17}$

In contrast, when a person is depressed, their immune system may also be depressed; this was found to be the case in a study of HIV positive men with depression. Those who were depressed were significantly more likely to have serological evidence of HIV infection and die from AIDS than those who were not depressed. ${ }^{18}$

Even when potentially stressful situations cannot be avoided, the effects can often be mitigated. In one study, researchers studied caregivers of children with autism and found that the more stress the women reported feeling, the shorter were the length of their telomeres. Women with the highest levels of perceived stress had significantly shorter telomeres, corresponding to a 9- to 17-year reduction in their life span. But the researchers found that it wasn't an objective measure of stress that determined its effects on their telomeres. It was how they reacted to the stress. Their perceptions of stress were more important than what was objectively occurring in their lives. ${ }^{19}$ Although these women were in very similar life situations, they had dramatically different outcomes. Those who made the healthy lifestyle choices described here were able to buffer the stress, so it didn't affect their telomeres and health: they became more resilient. In contrast, the others showed a significant shortening of their telomeres and, as such, their lives.

This is a very empowering finding: even when the external environment cannot be changed, how a person reacts can be modified: not to blame but to empower.

\section{Eat well}

Numerous studies have clearly shown that a healthy whole foods, plant-based diet enhances your immune function. There are thousands of substances in fruits, vegetables, whole grains, legumes and soy products that help protect against infections and suppress inflammatory markers. ${ }^{20-22}$ These include phytochemicals, bioflavonoids, carotenoids, retinols, isoflavones, genistein, lycopene and others.

Other nutrients that have been identified as critical for the growth and function of immune cells include vitamin C, vitamin $\mathrm{D}$, zinc, selenium, iron and proteins (including the amino acid glutamine). ${ }^{23}$ Diets that are limited in variety and lower in nutrients (consisting primarily of ultra-processed foods and, to a lesser extent, minimally processed foods) can negatively affect a healthy immune system. A western diet (high in processed foods, refined carbohydrates and animal protein (especially red meat), and low 
in fruits and vegetables) can affect the microbiome and suppress immunity. ${ }^{24}$

\section{Move more}

Many studies have shown a clear, inverse relationship between moderate exercise and risk of a wide variety of illnesses, including infectious diseases. Exercise enhances immune regulation. Although there has been some controversy that intense exercise may depress immune function, a review of the science indicates that frequent exercise enhances (rather than suppresses) immune competency. ${ }^{25}$ Even moderate exercise (eg walking 15-30 minutes/day) enhances immune function. ${ }^{26}$

In summary, the lifestyle changes described here will help prevent and may even reverse the progression of the same chronic diseases that increase the risk of contracting and dying from COVID-19. Enhancing our immune system can also help us to be less 'influenced' by the influenza virus.

\section{Putting this evidence into practice in the UK}

There is a wealth of evidence that lifestyle interventions can have a positive effect on health, but this is not always put into practice. The 'inverse health law' describes how those who might benefit most from lifestyle interventions are the least likely to do so. There are a variety of reasons for this, ranging from lack of knowledge, health literacy or resources to health simply not being a priority for those living the most difficult lives.

Take social isolation as an example. The former US surgeon general, Vivek Murthy, has described the 'paradox of loneliness', which leads to the loneliest becoming the most resistant to social intervention. It has been estimated, for instance, that lonely brains detect social threat twice as fast as non-lonely ones. ${ }^{27} \mathrm{How}$, then, do we overcome such inbuilt resistance to improving our lifestyle?

\section{The role of government in implementing lifestyle interventions}

There is much that government can and needs to do after the pandemic state of emergency. Addressing the appalling inequalities in wealth and opportunity in modern Britain could be a start. These inequalities can be seen in the large and increasing differences in life expectancy between rich and poor. ${ }^{28}$ More specifically, deprived areas in the UK see 20,000 more cancer cases each year than wealthier parts of the country. ${ }^{29}$ Specific action will need to include subsidising healthy food choices and enabling improved access to exercise facilities and green areas. The government can only achieve so much. A much more fundamental change in individual behaviour and local culture will be required if we are to create the underswell needed to bring about sustainable improvement in our lifestyles. Does medicine have a role in this? Absolutely it does!

\section{The role of social and healthcare systems in implementing lifestyle interventions}

Medicine will only become relevant to these issues when all clinicians and local people are encouraged and enabled to play a proper role in the health of their communities; as doctors, we will need to step out of our consulting rooms and on to the streets. Only by so doing can we fulfil the rhetoric of the NHS becoming a 'health' rather than a 'disease' service.
Social prescribing (SP) is opening the door to these possibilities. It has three elements. First, there is the referral by the general practitioner (GP) and, now increasingly, by other primary and secondary care clinicians and also self-referral by patients themselves. That referral is made to a SP link worker who sees the patient and can then connect the patient to a wide range of non-medical options. Since July 2019, SP link workers have been allotted to groups of GP practices with the aim that each group will have two or three within a short time. The SP link worker seeks to address those inequalities that we have been describing by seeing those who are least able and, sometimes, least willing initially to make the lifestyle changes that may improve their health. The initial intervention is about forming a relationship and understanding the patient's perspective, and this may lead to a whole range of secondary interventions from benefits help, housing and employment to physical exercise, arts and social opportunities. Restoring a sense of purpose in this way produces all sorts of beneficial effects and many have become local community champions. Research in Croydon, south-west London, Gloucestershire and Rotherham has shown a consistent reduction in use of hospitals and family doctor services by those referred to a link worker but the potential benefits to personal and community health could prove to be even more significant. ${ }^{30}$

SP is also creating ripple effects in our thinking about how we improve local health. Not only is it de-medicalising the medics, it is also mobilising patients and people. In Mendip, Somerset, for instance, it has led to the creation of 1,000 community connectors (taxi drivers, hairdressers, supermarket staff and students) each carrying a badge and playing an active community role in connecting those that are difficult to reach (such as the lonely and the isolated) to interventions that might improve their health and wellbeing. In Ilfracombe, Devon, there is a volunteer care facilitator on every street ensuring that the right people get the right voluntary help. In Bexley and elsewhere, Altogether Better have created an army of 25,000 volunteer champions enabling their peers to live healthier lives. ${ }^{31}$

In many areas, surprisingly, COVID 19 has hastened the process. Witness the 750,000 NHS volunteers, link workers extending their role from receiving referrals to case finding themselves, and the new effective working relationships in some areas between local authorities, voluntary sector and primary care. ${ }^{32}$

\section{Conclusion}

If we are serious about improving lifestyle (especially for those for whom the benefit is greatest) then we need a change in the current social order, which enabled the creation of these unhealthy lifestyles in the first place. This will require a new social movement (a social revolution, no less) that redefines the relationship between patient and clinician, between the individual and the community and between the NHS and all health-related areas in the community. It will challenge a risk-averse medical culture that has historically failed to enable patients and the community to become the engineers of healthier lifestyles. There may be gaps in the current evidence base (as with any fast-moving social movement) but these will surely be more than compensated with faith, courage and a renewal of medical ideals tempered with a little common sense? COVID-19 has revealed the deep flaws in our current system, but it has also shown what can be achieved when we are innovative, determined and prepared to make compromises. If we want to radically change the nation's lifestyle 
then it is time to be bold and ambitious. In the words of Robert Browning, future medicine's 'reach should exceed [its] grasp'.

\section{References}

1 The New York Times. United Kingdom coronavirus map and case count. The New York Times, 2021. www.nytimes.com/interactive/ 2020/world/europe/united-kingdom-coronavirus-cases.htm [Accessed 01 February 2021].

2 Search term: covid cases worldwide. Google. www.google.comsearch? $q=$ covid+cases+worldwide\&oq =covid+cases+worldwide\&aqs= chrome..69i57 j0l2j0i39517.274 4j1j7\&sourceid=chrome\&ie=UTF-8 [Accessed 01 February 2021].

3 Powell A. Fauci says herd immunity possible by fall, 'normality' by end of 2021. The Harvard Gazette 2020. https://news.harvard.edu/ gazette/story/2020/12/anthony-fauci-offers-a-timeline-for-endingcovid-19-pandemic [Accessed 01 February 2021].

4 Xia Y, Li Q, Li W, Shen H. Elevated mortality of chronic diseases during COVID-19 pandemic: a cause for concern? Ther Adv Chronic Dis 2020;11:2040622320961590.

5 Ornish D, Ornish A. UnDo It! How simple lifestyle changes can reverse most chronic diseases. New York: Random House, 2019.

6 Oldways common ground. Oldways common ground. Oldways. https://oldwayspt.org/programs/oldways-common-ground/ oldways-common-ground.

7 Warburton DER, Bredin SSD. Health benefits of physical activity: a systematic review of current systematic reviews. Curr Opin Cardio 2017:32:541-56

8 Creswell JD, Irwin MR, Burklund LJ et al. Mindfulness-based stress reduction training reduces loneliness and pro-inflammatory gene expression in older adults: a small randomized controlled trial. Brain Behav Immun 2012;26:1095-101.

9 Ornish D. Love \& survival: the scientific basis for the healing power of intimacy. New York: HarperCollins, 1998.

10 Ford ES, Bergmann MM, Kröger ] et al. Healthy living is the best revenge: findings from the European prospective investigation into cancer and nutrition: Potsdam study. Arch Intern Med 2009:169:1355-62.

11 Li Y, Pan A, Wang DD, Liu X et al. Impact of healthy lifestyle factors on life expectancies in the US population. Circulation. 2018;138:345-55.

12 Biondi M, Zannino LG. Psychological stress, neuroimmunomodulation, and susceptibility to infectious diseases in animals and man: a review. Psychother Psychosom 1997;66:3-26.

13 Murthy VH. Together: The Healing power of human connection in a sometimes lonely world. New York: Harper, 2020.

14 Segerstrom SC, Miller GE. Psychological stress and the human immune system: a meta-analytic study of 30 years of inquiry. Psychol Bull 2004;130:601-30.

15 Cohen S, Doyle WJ, Skoner D et al. Social ties and susceptibility to the common cold. JAMA 1997;277:1940-4.

16 Cohen S, Tyrrell DAJ, Smith AP. Psyschological stress and susceptibility to the common cold. N Engl ] Med 1991;325:606-12.
17 Cohen S, Doyle WJ, Turner RB et al. Emotional style and susceptibility to the common cold. Psychosomatic Medicine 2003:65:652-7.

18 Mayne TJ, Vittinghoff E, Chesney MA et al. Depressive affect and survival among gay and bisexual men infected with HIV. Arch Intern Med 1996;156:2233-8.

19 Epel ES, Blackburn EH, Lin ] et al. Accelerated telomere shortening in response to life stress. Proc Natl Acad Sci USA 2004;101:17312-5.

20 Lampe JW. Spicing up a vegetarian diet: chemopreventive effects of phytochemicals. Am J Clin Nutr 2003;78(3 Suppl):579S-83S.

21 Childs CE, Caler PC, Miles EA. Diet and immune function. Nutrients 2019;11:1933.

22 Craddock JC, Neale EP, Peoples GE et al. Vegetarian-based dietary patterns and their relation with inflammatory and immune biomarkers: a systematic review and meta-analysis. Adv Nutr 2019;10:433-51.

23 Harvard TH Chan School of Public Health. Nutrition and immunity. Harvard TH Chan School of Public Health. www.hsph.harvard.edu/ nutritionsource/nutrition-and-immunity [Accessed 01 February 2021].

24 Molendijk I, van der Marel S, Maljaars PW. Towards a Food pharmacy: immunologic modulation through diet. Nutrients. 2019;11:1239.

25 Campbell JP. Turner JE. Debunking the myth of exercise-induced immune suppression: redefining the impact of exercise on immunological health across the lifespan. Front Immunol 2018;9:648.

26 Nieman DC, Wentz LM. The compelling link between physical activity and the body's defense system. Journal of Sport and Human Science 2019:8:201-17.

27 Cacioppo S, Bangee M, Balogh S et al. Loneliness and implicit attention to social threat. Cognitive Neuroscience 2016;7:138-59.

28 The Health Foundation. Astonishing difference in the risk of avoidable death between the rich and poor. The Health Foundation, 2019. www.health.org.uk/news-and-comment/news/astonishingdifference-in-the-risk-of-avoidable-death

29 Cancer Research UK. Cancer in the UK 2020: Socio-economic deprivation. Cancer Research UK, 2020. www.cancerresearchuk.org/ sites/default/files/cancer_inequalities_in_the_uk.pdf

30 NHS England. Recruiting and managing volunteers in NHS pro viders: a practical guide. NHS, 2017

31 Altogether Better. Citizens. Altogether Better. www.altogetherbetter. org.uk/citizens

32 Polley M, Bertotti M, Kimberlee R, Pilkington K, Refsum C. A review of the evidence assessing impact of social prescribing on healthcare demand and cost implications. University of Westminster, 2017.

33 Browning R. Men and women. Boston: Ticknor and Fields, 1855.

Address for correspondence: Dr Michael Dixon, College of Medicine and Integrated Health, 9th floor, Borough Wing, Guy's Hospital, Great Maze Pond, London SE1 9RT, UK. Email: michaeldixon@nhs.net 